\title{
Comparing the Effectiveness of the Muscle Energy Technique and Kinesiotaping in Mechanical Sacroiliac Joint Dysfunction: A Non-blinded, Two-Group, Pretest-Posttest Randomized Clinical Trial Protocol
}

\author{
Manisha Sarkar ${ }^{1}$, Manu Goyal ${ }^{1}$, Asir John Samuel ${ }^{2}$ \\ ${ }^{1}$ Department of Musculoskeletal Physiotherapy, Maharishi Markandeshwar Institute of Physiotherapy and Rehabilitation, Maharishi Markandeshwar \\ (Deemed to be University), Ambala, India \\ ${ }^{2}$ Department of Pediatric and Neonatal Physiotherapy, Maharishi Markandeshwar Institute of Physiotherapy and Rehabilitation, Maharishi \\ Markandeshwar (Deemed to be University), Ambala, India
}

Study Design: Two-group, pretest-posttest randomized clinical trial.

Purpose: To evaluate the efficacy of the muscle energy technique (MET) and Kinesiotaping (KT) in addition to conventional physiotherapy among patients with mechanical sacroiliac joint dysfunction (SIJD).

Overview of Literature: Patients with SIJD suffer from lower back and gluteal pain, as well as stiffness, due to restricted pelvic joint movement. To restore function and reduce pain among individuals with mechanical SIJD, the MET and KT may be helpful. However, a limited number of studies have compared MET and KT in mechanical SIJD.

Methods: A total of 40 male and female participants aging between 30 and 50 years experiencing unilateral pain around the gluteal area, groin area, and lower limbs for more than 4 weeks but less than 1 year will be selected. Patients will then be randomized into two groups: METCp ( $\mathrm{n}=20)$ and KTCp groups $(\mathrm{n}=20)$. The METCp group will receive MET along with conventional physiotherapy, while the KTCp group will receive conventional physiotherapy with KT. Both groups will receive three alternating days of treatment per week that will continue for 4 weeks. The modified Oswestry Disability Index, a digital pressure algometer, and sacroiliac joint motion testing will be used for evaluation. Between- and within-group pre- and post-intervention results for mechanical SIJD were compared using the Wilcoxon signed-rank test/paired $t$-test and Mann-Whitney $U$-test/independent $t$-test.

Results: Modified Oswestry Disability Index, digital pressure algometer, and sacroiliac joint motion will be measured at baseline, 2nd week during intervention, and 4th week at the end of intervention

Conclusions: The present study will provide data regarding the effects of MET and KT among patients with mechanical SIJD.

Keywords: Athletic tape; Low back pain; Manipulation; Pelvic girdle pain; Orthotic tape; Muscle energy technique

Received Sep 10, 2019; Revised Oct 2, 2019; Accepted Oct 6, 2019

Corresponding author: Asir John Samuel

Department of Pediatric and Neonatal Physiotherapy, Maharishi Markandeshwar Institute of Physiotherapy and Rehabilitation, Maharishi Markandeshwar (Deemed to be University), Mullana, Ambala, Haryana, India

Tel: +91-8059930222, E-mail: asirjohnsamuel@mmumullana.org 


\section{Introduction}

Sacroiliac joint dysfunction (SIJD) is the most common cause for nondiscogenic pain. Mechanical SIJD is mainly characterized by pain in the sacroiliac joint (SIJ) area due to stiffness and decreased mobility of SIJ, also called hypomobility. The pain becomes pronounced while standing up from seated position, standing, walking, and lifting objects and is felt on same side (unilateral) at which the SIJ is involved [1]. The SIJ, which bears approximately $60 \%$ of the body weight, is involved in several painful conditions related to the pelvis and lower extremities. SIJD, which may lead to biomechanical alterations in the structures around the pelvis [2], is the most common source of lower back pain affecting $70 \%-85 \%$ of adults. Accordingly, $13 \%-30 \%$ of patients with lower back pain have SIJD, while $72.3 \%$ of those with lumber disc herniation have the same [3].

SIJD has been mainly associated with pain and hypoor hypermobility. Several studies have explored different treatment options for SIJD. Accordingly, Laslett [4] found that manual therapy with lumbopelvic stabilization is a promising technique that helps improve overall quality of life. Another study by Shinde and Jagtap [2] revealed significant improvements in the muscle energy technique (MET), hot moist pack, mulligan mobilization, and mulligan taping groups. Al-Subahi et al. [5] also reported that manipulation, exercise, and Kinesiotaping (KT) were effective in treating pain, disability, and pelvic asymmetry in SIJD. Another study by Yoo [6] showed that gluteus medius strengthening exercises can help reduce SIJ pain.

The MET includes soft tissue manipulation methods consisting of controlled isometric and/or isotonic contractions designed to improve normal physiologic function and decrease pain. The MET can be described as forces exerted by the patient against the therapist's counter force, which could results in maximum muscle contraction [2]. KT has also been used to reduce pain and stabilize the alternating structures associated with SIJD [7]. Although MET can be an effective treatment for SIJD, several studies have reported that $\mathrm{KT}$ alone does not have much effect on SIJ pain. Hence, the present study seeks to compare the effects of KT and MET in addition to conventional physiotherapy among patients with mechanical SIJD.

\section{Materials and Methods}

\section{Research design and ethics}

This is a parallel design, non-blinded, two-group, pretestposttest randomized clinical trial involving patients with mechanical SIJD. The protocol was approved by the Ethics Research Committee of the Maharishi Markandeshwar (Deemed to be University) (protocol ID: MMDU/ IEC/1370). The study has been registered successfully in the World Health Organization International Clinical Trials Registry (NCT03940287) on 4 May 2019 and obtained its universal trial number (U111-1232-1305) on 16 April 2019. The study was performed in accordance with the Indian Council of Medical Research (2017) National Ethical Guidelines for biomedical and health research involving human participants and the ethical principles for medical research involving human subjects stated in the Declaration of Helsinki (revised 2013).

\section{Participants}

Participants from the recognized tertiary teaching center at Mullana, Ambala, Haryana will be recruited between August 2019 and March 2020 (data collection between October 2019 and February 2020). All participants will be asked to sign an informed consent before the intervention that shall be provided in the local language with which the patient is comfortable. Confidentiality will be ensured by assigning codes for data collection, with only the primary researcher holding key to participant codes in each group.

\section{Eligibility criteria}

According to the International Association for the Study of Pain criteria [8], the inclusion criteria will be as follows: both male and female patients aging between 30 and 50 years, pain in the lower back, gluteal and groin area, and lower extremity lasting for more than 4 weeks but less than 1 year [5], restricted SIJ motion determined through clinical testing, and apparent leg length discrepancy.

Patients with any medical conditions that would make exercise difficult, such as pelvic bone fractures, pelvic metallic implants (endoprostheses), SIJ inflammation (e.g., sacroiliitis), somatoform disorders, pregnancy, inflammatory bowel disease, and malignancy, will be excluded [9] 


\section{Randomization, allocation, and blinding}

A total of 40 eligible participants will be randomized to the MET and conventional physiotherapy group (METCp group) and $\mathrm{KT}$ and conventional physiotherapy group (KTCp group) via block randomization using the sequentially numbered opaque sealed envelope method. Blocks will have even numbers with a $4 \times 10$ (40) matrix, suggesting a total of four blocks with 10 rows. Subjects will then be allocated into each block using a random allocation sequence. Thereafter, subjects will be allocated to experimental 1 group (METCp) and experimental 2 group (KTCp). No blinding was done in this study given that all procedures and outcome measures will be conducted and assessed by therapist herself. The schematic Consolidated Standards of Reporting Trials flow chart for the study protocol (MET-KET) is displayed in Fig. 1.

\section{Enrolment and baseline measurements}

Baseline characteristics of each patient will be determined by the physiotherapist through standardized assessment pro forma. All physical examinations will be performed before proceeding with the intervention. Measures such as demographic profile (age, gender, height, weight and medical history), socioeconomic status, personal habits, history of present illness, and pain history and comorbidities will be evaluated in the assessment pro forma for mechanical SIJD, after which pain associated with SIJD (digital pressure algometer), muscle strength (hand-held dynamometer), quality of life (modified Oswestry Disability Index) will be evaluated. Patient participation schedules for the trial will be determined in accordance with the guidelines provided by the Standard Protocol Items: Recommendations for Interventional Trials statement [10] (Fig. 2).

\section{Sample size}

Sample size was calculated using the $\mathrm{G}^{\star}$ Power tool (Heinrich-Heine-Universität Düsseldorf, Düsseldorf, Germany; http://www.gpower.hhu.de/) for sample size calculations [11]. Digital pain pressure algometer values for studies related to SIJD was considered given that this is the primary outcome measure used herein. Mean post- (9.02) and preintervention (7.46) values were considered and further divided according to a standard deviation value

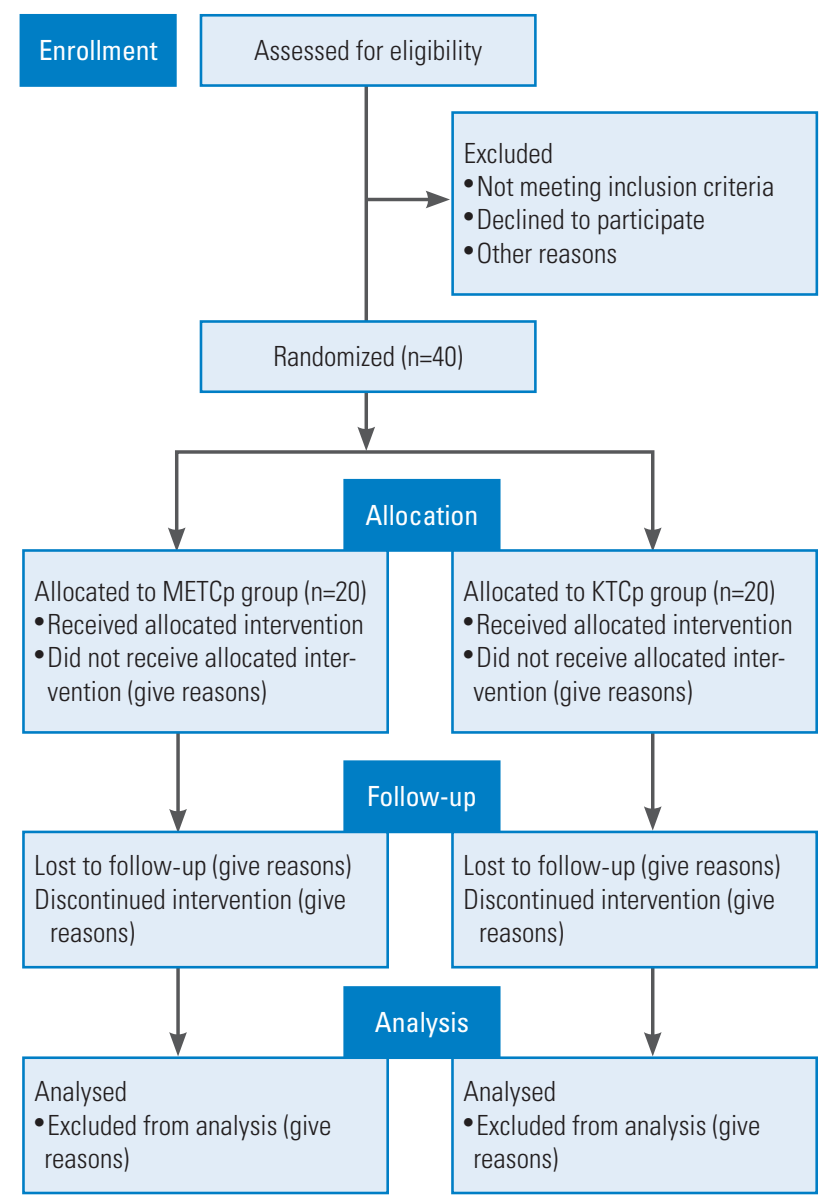

Fig. 1. Schematic Consolidated Standards of Reporting Trials flow chart for the study protocol (MET-KET). MET, muscle energy technique; KT, Kinesiotaping; METCp group, MET and conventional physiotherapy group; KTCp group, KT and conventional physiotherapy group.

of 1.62 to calculate the effect size $(0.96)[12,13]$. The level of significance will be set at 0.05 . To obtain a power of $90 \%(\beta=10 \%)$, a sample size of $\mathrm{n}=15$ in each group will be needed according to estimates using $G^{\star}$ Power ver. 3.1.9.4 software (Heinrich-Heine-Universität Düsseldorf) [14]. Moreover, considering a 30\% dropout rate, a final sample of $n=20$ in each group will be needed.

\section{Interventions}

After completing all assessments and obtaining baseline measures, the intervention will be conducted. Participant will be randomly assigned to the METCp group and the KTCp group. Each participant will undergo three sessions per week that will continue for 4 weeks. Schober's test will be used to determine the lumbar range of motion. First, patients will be undergo seated flexion and standing flexion tests to identify the SIJD, after which pain intensity 


\begin{tabular}{|c|c|c|c|c|c|c|c|c|}
\hline \multirow[b]{3}{*}{ Timepoint } & \multicolumn{8}{|c|}{ Study period } \\
\hline & \multirow{2}{*}{\begin{tabular}{|c|} 
Enrolment \\
0 wk
\end{tabular}} & \multirow{2}{*}{$\begin{array}{c}\text { Allocation } \\
0 \text { wk } \\
\end{array}$} & \multicolumn{4}{|c|}{ Post-allocation (treatment) } & \multicolumn{2}{|c|}{$\begin{array}{l}\text { Follow-up } \\
\text { (evaluations) }\end{array}$} \\
\hline & & & 1st wk & 2nd wk & 3rd wk & 4th wk & 2nd wk & 4th wk \\
\hline \multicolumn{9}{|l|}{ Enrolment } \\
\hline Eligibility screen & $x$ & & & & & & & \\
\hline Informed consent & $x$ & & & & & & & \\
\hline Clinical evaluation, inclusion and exclusion criteria & $x$ & & & & & & & \\
\hline Allocation & & $x$ & & & & & & \\
\hline \multicolumn{9}{|l|}{ Interventions } \\
\hline Muscle energy technique along with conventional physiotherapy & & & $x$ & $x$ & $x$ & $x$ & & \\
\hline Kinesiotaping along with conventional physiotherapy & & & $x$ & $x$ & $x$ & $x$ & & \\
\hline \multicolumn{9}{|l|}{ Assessments } \\
\hline Demographic data & & $x$ & & & & & & \\
\hline Pain pressure threshold (calibrated digitalized algometer) & & $x$ & & & & & $x$ & $x$ \\
\hline Hand held dynamometer (Hydraulic) & & $x$ & & & & & $x$ & $x$ \\
\hline Modified Oswestry Disability Index & & $x$ & & & & & $x$ & $X$ \\
\hline
\end{tabular}

Fig. 2. Standard Protocol Items: Recommendations for Interventional Trials schedule for patient participation.

will be measured using a calibrated digital pain pressure algometer (ALGO-DS-01). Static stabilizer strength of the SIJ and quality of life will then be measured using a handheld dynamometer (hydraulic) and the modified Oswestry Disability Index, respectively [15].

1) Muscle energy technique application

The METCp group will undergo the MET together with conventional treatment. MET will be applied for anterior and posterior innominate rotation around the pelvis and for weakened muscles like the piriformis. For anterior iliac rotation, patients will be placed in the prone position with the affected leg brought over the edge of the table by flexing the hip as shown in Fig. 3. Thereafter, one hand will be used to stabilize the sacral area, while the other hand will be used to support hip flexion and aid toward greater flexion until a barrier is sensed. The patient will then be asked to apply $20 \%$ force against that applied by the therapist and hold that contraction for 10 seconds over 5 to 12 repetitions. For posterior rotation, the patient will be placed in the prone position with the therapist standing opposite to the dysfunctional side as shown in Fig. 4. The affected leg will be then extended until free movement ceases with the patient exerting $20 \%$ of their strength and holding the contraction for 10 seconds over 5 to 12 repetitions [16]. For piriformis application, the patient will be placed in

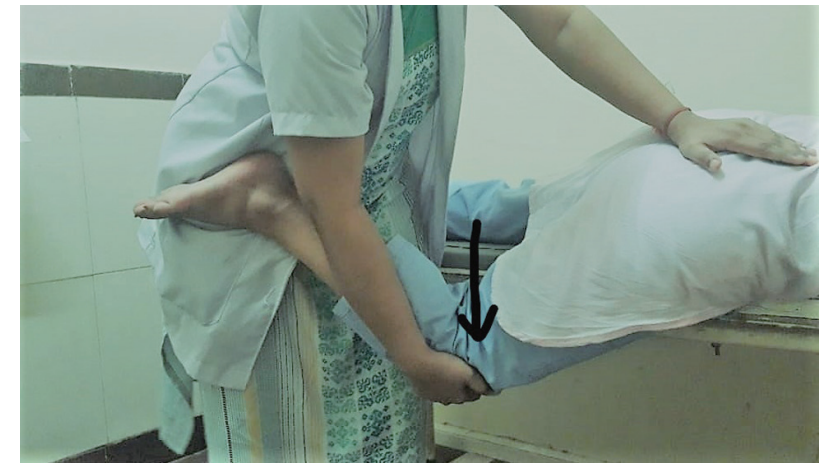

Fig. 3. Muscle energy technique for anterior innominate rotation (arrow).

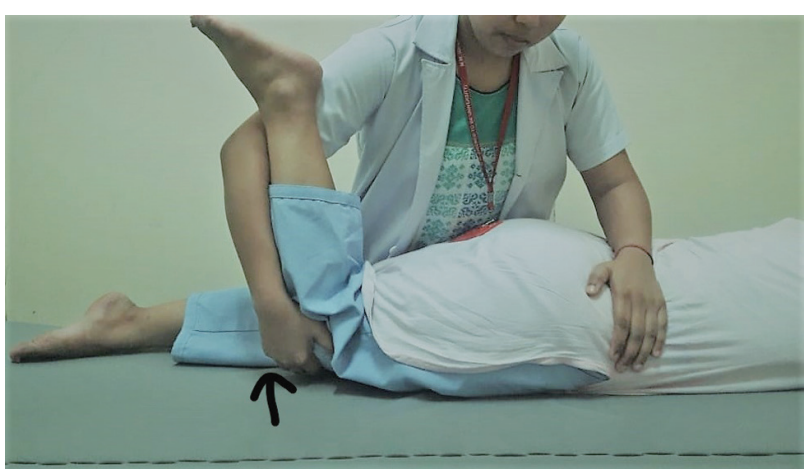

Fig. 4. Muscle energy technique for posterior innominate rotation (arrow).

the supine position close to the edge of the table with the affected side flexed at the hip and knee. The therapist will then stand facing the patient at the hip level as shown in 


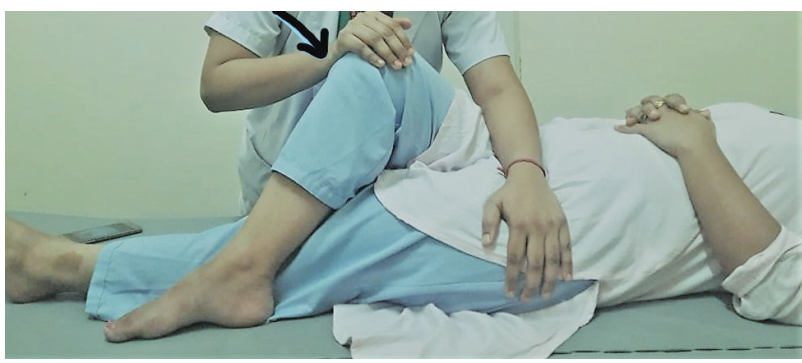

Fig. 5. Muscle energy technique for the piriformis muscle (arrow).
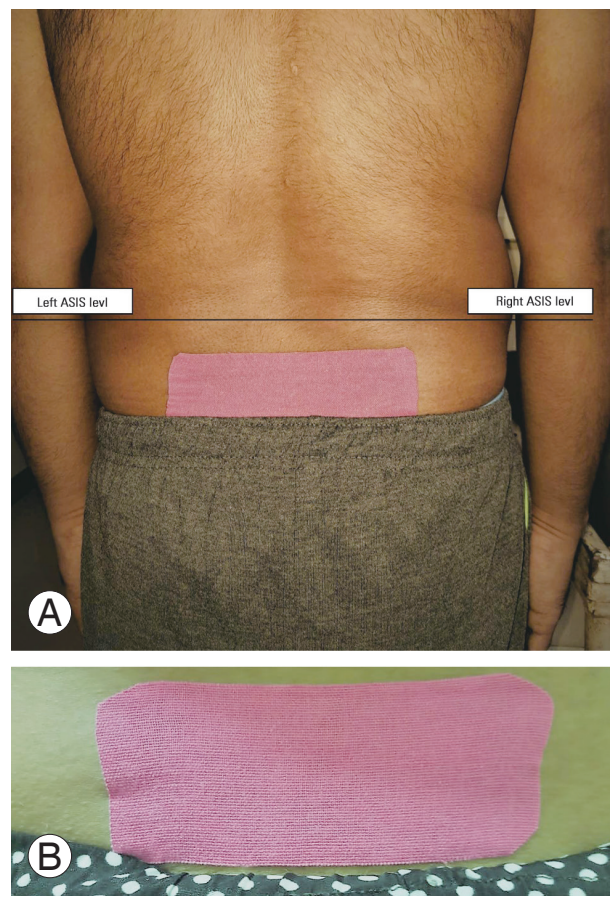

Fig. 6. (A, B) Kinesiotaping for the sacroiliac joint. ASIS, anterior superior iliac spine.

Fig. 5. The patient should be close enough to the edge of the table for the therapist to stabilize the pelvis with their hand. The angle of hip flexion should not exceed $60^{\circ}$. One hand is then placed on the contralateral anterior superior iliac spine to prevent pelvic motion, while the other hand is placed against the lateral flexed knee as it is pushed into resisted abduction to contract the piriformis for 7-12 seconds. Treatment will be provided on three alternating days per week that will continue for 4 weeks [17]. The MET of the protocol titled "Manisha's-muscle energy technique (M-MET) treatment protocol for mechanical sacroiliac joint dysfunction patients" by Manisha Sarkar, Manu Goyal, and Asir John Samuel was copyrighted under the Copyright Office of the Government of India with unique registration no. L-85162/2019 dated 3rd September, 2019 (copyright filed with diary no., 10909/2019-CO/L dated

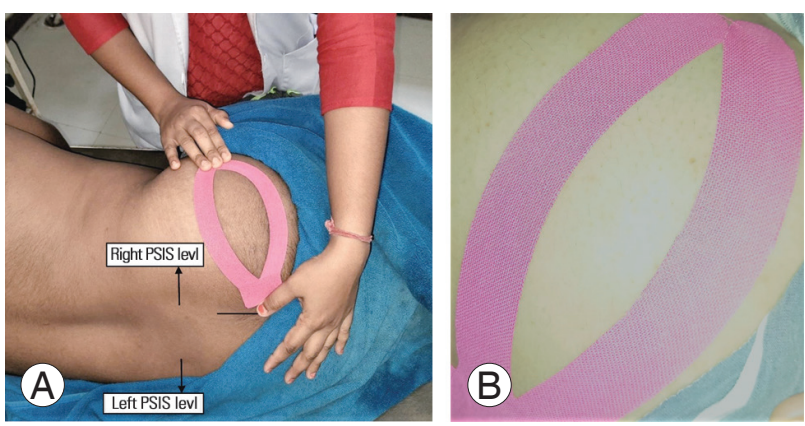

Fig. 7. (A, B) Kinesiotaping for the piriformis muscle. PSIS, posterior superior iliac spine.

15th July, 2019). The detailed description for M-MET is displayed in Table 1.

\section{2) Kinesiotaping application}

The KTCp group will receive KT together with conventional physiotherapy. Accordingly, KT will be applied to the piriformis muscle and SIJ. This tape will be applied in the shape of an "I" to both sides of the spine from the sacrum to the anterior superior iliac spine. For SIJ taping, the same 5-cm KT tape will be used and applied from below the posterior sacroiliac spine to the opposite side. Here, the patient will be placed in a standing position as shown in Fig. 6. For piriformis application, the patient will be placed in a side lying position 7 where the effected leg will be placed uppermost with hip in flexion, adduction, and internal rotation as displayed in Fig. 7. The therapist will position near the level of the pelvis, while the base of the tape will be placed over the contralateral part of the sacrum, forming a "Y" strip with minimum tension attached over the greater trochanter of the ipsilateral femur [18]. Patients will undergo KT treatment 3 times a week for 4 weeks to decrease pain and increase stability [7]. The kinesiotape application technique titled "Manisha's kinesiotape (M-KT) application protocol for patients with mechanical sacroiliac joint dysfunction" by Manisha Sarkar, Manu Goyal, and Asir John Samuel was copyrighted under the Copyright Office of the Government of India with unique registration no L-88904/2020 filed with diary no., 15442/2019-CO/L dated 30th September, 2019. A detailed description of the $\mathrm{KT}$ application (M-KT) is provided in Table 2.

\section{3) Conventional physiotherapy}

Both groups will undergo conventional physiotherapy. Before undergoing the intervention, both groups will be given hydrocollator packs for at least 5-15 minutes [19]. 


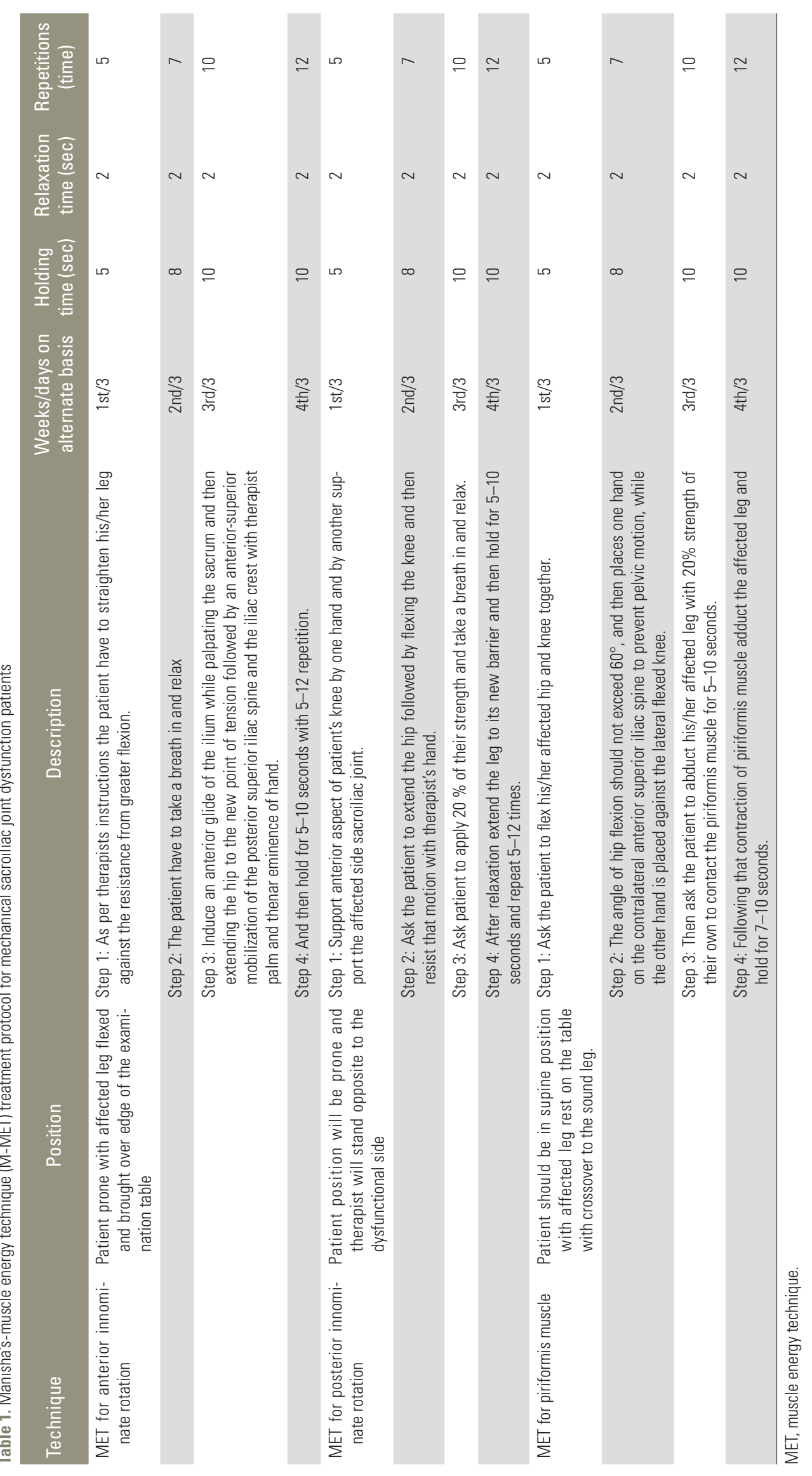


Table 2. Manisha's kinesiotape (M-KT) application protocol for patients with mechanical sacroiliac joint dysfunction

\begin{tabular}{|c|c|c|c|}
\hline $\begin{array}{l}\text { Area of KT tape } \\
\text { application }\end{array}$ & Description & Stretch force applied & $\begin{array}{l}\text { Weeks/days on } \\
\text { alternate basis }\end{array}$ \\
\hline \multirow[t]{4}{*}{ Piriformis muscle } & $\begin{array}{l}\text { Step 1: Patient position should be in side lying with the affected leg placed uppermost } \\
\text { with hip in flexion, adduction, and internal rotation. }\end{array}$ & Approximately 10\%-20\% & $1 \mathrm{st} / 3$ \\
\hline & Step 2: Therapist position should be in standing position near the level of pelvis. & Approximately $10 \%-20 \%$ & $2 n d / 3$ \\
\hline & Step 3: Take any available colour KT tape with 5-cm length \& cut it down in ' $Y$ ' shape. & Approximately 10\%-20\% & $3 \mathrm{rd} / 3$ \\
\hline & $\begin{array}{l}\text { Step 4: Then KT tape should be pulled apart to both the side from middle part of its } \\
\text { length. } \\
\text { Step 5: Then place the base of KT tape at the sacrum of affected side and then by ap- } \\
\text { plying } 20 \% \text { stretch two anchors have to be placed along the course of the muscle \& } \\
\text { ended at the greater trochanter of femur. }\end{array}$ & Approximately $10 \%-20 \%$ & 4 th/3 \\
\hline \multirow[t]{4}{*}{ Sacroiliac joint } & Step 1: Patient should be in lean forward while standing. & Approximately $10 \%-20 \%$ & $1 \mathrm{st} / 3$ \\
\hline & Step 2: Take any available colour KT tape with 5-cm length \& cut it down in 'I' shape. & Approximately $10 \%-20 \%$ & $2 \mathrm{nd} / 3$ \\
\hline & Step 3: Then KT tape should be pulled apart to both the side of the anchor. & Approximately 10\%-20\% & $3 \mathrm{rd} / 3$ \\
\hline & $\begin{array}{l}\text { Step 4: Then place the middle part of KT tape by applying total } 10 \%-20 \% \text { stretch } \\
\text { below the posterior spine to both opposite side. } \\
\text { Step 5: After that apply the two anchors with zero stretch. }\end{array}$ & Approximately $10 \%-20 \%$ & 4 th $/ 3$ \\
\hline
\end{tabular}

KT, Kinesiotape.

\section{Outcome measures}

All outcomes will be assessed at baseline and 2 and 4 weeks after the intervention, with a follow-up assessment 4 weeks thereafter. All instruments used for assessment during the intervention should have good validity and reliability. Here, the primary outcome measures will be assessed using a digital pain pressure algometer and handheld dynamometer, while secondary outcome measures will be assessed using the modified Oswestry Disability Index.

\section{1) Pain intensity}

Pain intensity, which is associated with SIJD, will be measured using a calibrated digital pain pressure algometer (ALGO-DS-01) in patients with mechanical SIJD. The digital algometer is a computer-sized force gage that is highly accurate. The ALGO-DS-01 was selected for the assessment of pain among patients with mechanical SIJD given its reliability and validity as a tool for assessing SIJD. Inter-rater reliability for the pressure pain algometer was calculated using the intraclass correlation coefficient, with results showing a moderate to good response (0.62-0.84) [20].

\section{2) Muscle strength}

Piriformis muscle strength will be evaluated by measuring the maximum isometric voluntary contraction using a (hydraulic) hand-held dynamometer, which has better validity than manual muscle testing and an isokinetic dynamometer. Moreover, a hand-held dynamometer is portable and more easy to operate [21].

\section{3) Quality of life}

Quality of life will be measured by using the modified Oswestry Disability Index in individuals with mechanical SIJD. This is an extremely important tool used to measure a patient's permanent functional disability. The total score ranges from 0 to 50, with each section having a total possible score of 5 . Patient can be further subcategorized into five groups according to their total score: $0 \%-20 \%$ (minimal disability), 20\%-41\% (moderate disability), 41\%-60\% (severe disability), 61\%-80\% (crippled), and 81\%-100\% (bedridden). The modified Oswestry Disability Index has a minimum change of $15.5 \%$. This measure has been proven to have good validity for detecting changes in the SIJ, with a minimum difference of 13-15 indicating clinical significance [22].

\section{Data analysis}

All data will be collected and analyzed by the primary researcher. Baseline characteristics of eligible participants will be presented using descriptive statistics. Normality of the collected data will be established using the ShapiroWilk test. Based on data normality, descriptive statistics will be expressed as mean \pm standard deviation or median 
and intra-quartile range. Within-group comparisons will be conducted using paired $t$-tests or the Wilcoxon signedrank test, while and between-groups comparisons will be conducted using independent $t$-test or the Mann-Whitney $U$-test. The level of significance was set at 0.05 for all analyses. Statistical analysis will be performed using the IBM SPSS statistical software ver. 22.0. (IBM Corp., Armonk, NY, USA).

\section{1) Data management}

Data will be entered into Microsoft Office Excel 2010 (Microsoft Corp., Redmond, WA, USA) and stored in a desktop without an internet connection to help prevent unauthorized data access. The final data will also be restored on a password-protected non-rewritable compact disc read only memory, with only the primary researcher and data analysts having knowledge of the password. Data will be overseen by the chairman of Student Project Committee, Maharishi Markandeshwar Institute of Physiotherapy and Rehabilitation, Maharishi Markandeshwar (Deemed to be a University), Mullana.

\section{2) Data and Safety Monitoring Committee}

Safety data will be overseen by the Data and Safety Monitoring Committee, which is under the oversight of the chairman of the Student Project Committee of Maharishi Markandeshwar Institute of Physiotherapy and Rehabilitation, Maharishi Markandeshwar (Deemed to be University), Mullana. This committee is independent from the primary researcher and plays a role in monitoring the outcome of the intervention. Any adverse results occurring during the study will prompt interim analysis.

\section{Results}

Outcome measures for pain intensity, piriformis muscle strength, and quality of life in the patient with mechanical SIJD will be measured at baseline, 2nd week during intervention, and 4 th week following intervention by, digital pain pressure algometer, (hydraulic) hand-held dynamometer, and modified Oswestry Disability Index, respectively.

\section{Discussion}

In the present study, individuals with mechanical SIJD will undergo the MET and KT in addition to conventional physiotherapy. Studies have shown that the MET, which mainly involves various neurological mechanisms with stretch tissue tolerance, and KT, which exerts immediate therapeutic effects, help support the stability of normal physiologic mechanisms and decrease anterior pelvic tilting [23]. Another study showed that pelvic tilt taping had an immediate effect on individuals with SIJ pain [24]. Moreover, the MET, along with other techniques, has been shown to help restore pubic symphysis alignment among patient with of pelvis asymmetry [5,25], suggesting that the MET is effective in managing SIJ pain. However, no study has yet compared the effects of MET and KT in addition to conventional physiotherapy in patients with mechanical SIJD. The main mechanism for developing SIJD includes different unidirectional pelvic shear forces along with repetitive torsional forces and inflammation, which produces not only the lower back pain but also groin and gluteal pain. A single-blind, prospective, randomized control trial by Visser et al. [26], suggested that manual therapy should be the main treatment for SIJ-related leg pain apart from other physiotherapy protocols. Orakifar et al. [27] also found that manual therapy and exercise therapy were two common treatments for chronic back pain associated with SIJD. Accordingly, their study they found that manual therapy and stabilization exercises had similar effects on reducing pain and improving quality of life [27]. Unilateral inclination can be assessed using pelvic landmarks. To determine the innominate torsional asymmetry in SIJD, four clinical tests have been used, including the seated and standing flexion tests [28]. However, randomized clinical trials, which would be of considerable help in investigating SIJD, will need to consider a single component to address. Thus, the main objectives of the present study will be to apply the MET and KT in addition to conventional physiotherapy in order to discover various physiological changes in pain perception and muscle strength among individuals who suffering from SIJD. The main limitations of this study will be the allocation of participants from the same rehabilitation center considering its single-center design.

Given the very limited number of studies comparing the MET and KT, the present study will seek to compare the effects of both techniques among individuals with mechanical SIJD. 


\section{Conclusions}

This protocol details the single-center clinical trial comparing the effects of MET and KT in addition to conventional physiotherapy on improving pain and quality of life among patients with mechanical SIJD.

\section{Conflict of Interest}

No potential conflict of interest relevant to this article was reported.

\section{Acknowledgments}

The author acknowledges the Maharishi Markandeswar Institute of Physiotherapy and Rehabilitation, Maharishi Markandeswar (Deemed to be University) for the precious support and encouragement throughout the study. This study was supported by the Maharishi Markandeshwar (Deemed to be University) PG Thesis Grant (grant no., MMDU/18188014/2018). The study is being supervised by the third author, Asir John Samuel and co-supervised by, the second author, Manu Goyal. Related to this study protocol, two copyrights were registered with unique registration nos. L-85162/2019 and L-88904/2020 under the Copyright Office of the Government of India.

\section{ORCID}

Manisha Sarkar: https://orcid.org/0000-0001-6090-0041

Manu Goyal: https://orcid.org/0000-0003-1618-1320

Asir John Samuel: https://orcid.org/0000-0003-1747-0415

\section{Author Contributions}

All the authors reconceived and designed the study, conducted research, provided research materials. MS drafted the manuscript. MG and AJS provided the content review. All the authors approved the manuscript.

\section{References}

1. Cusi MF. Paradigm for assessment and treatment of SIJ mechanical dysfunction. J Bodyw Mov Ther 2010;14:152-61.

2. Shinde M, Jagtap V. Effect of muscle energy technique and mulligan mobilization in sacroiliac joint dysfunction. Glob J Res Anal 2018;7:79-81.

3. Kunene SH, Luthuli H, Nkosi M, Haffejee M, Jooma I, Munro S. Mechanical lower back pain and sacroiliac joint dysfunction in golfers at two golf clubs in Durban, South Africa. S Afr J Physiother 2018;74:402.

4. Laslett M. Evidence-based diagnosis and treatment of the painful sacroiliac joint. J Man Manip Ther 2008;16:142-52.

5. Al-Subahi M, Alayat M, Alshehri MA, et al. The effectiveness of physiotherapy interventions for sacroiliac joint dysfunction: a systematic review. J Phys Ther Sci 2017;29:1689-94.

6. Yoo WG. Effects of individual strengthening exercises on subdivisions of the gluteus medius in a patient with sacroiliac joint pain. J Phys Ther Sci 2014;26:1501-2.

7. Oh HJ, Jeon CB, Jeong MG, Choi SJ. The effects of spinal decompression therapy on pain and disability in patients with chronic low back pain. J Korean Phys Ther 2017;29:299-302.

8. Kenkampha K, Boonprakob DY, Arayawichanon P. Sacroiliac joint mobilization immediate improved clinical features of non-specific low back pain with sacroiliac joint dysfunction. Khon Kaen Univ Res J (Grad Stud) 2013;13:71-84.

9. Bindra S. A study on the efficacy of muscle energy technique as compared to conventional therapy on lumbar spine range of motion in chronic low back pain of sacroiliac origin. Hum Biol Rev 2013;2:33648.

10. SPIRIT (Standard Protocol Items: Recommendations for Interventional Trials) [Internet]. [place unknown]: The SPIRIT Group [cited 2019 Oct 2]. Available from: https://www.spirit-statement.org/.

11. Peacock J, Kerry S. Writing a research protocol. In: Peacock J, Kerry S, editors. Presenting medical statistics from proposal to publication. 2nd ed. Oxford: Oxford University Press; 2007. p. 16-7.

12. Portney LG, Watkins MP. Statistical measures of validity. In: Portney LG, Watkins MP, editors. Foundations of clinical research: applications to practice. 3rd ed. Philadelphia (PA): F. A. Davis Company; 2015. p. 645-6.

13. Sabour AA, Awad MA, Mansy AA, Tolba M. Effect of mulligan technique on sacroiliac dysfunction during pregnancy. Med J Cairo Univ 2016;84:35-41.

14. Faul F, Erdfelder E, Lang AG, Buchner A. G*Power 
3: a flexible statistical power analysis program for the social, behavioral, and biomedical sciences. Behav Res Methods 2007;39:175-91.

15. Verma CV, Renuka D, Vijaya K, Amol B. Lumbar range of motion: reliability between Schober's test \& modified Schober's test. Rom J Phys Ther 2015;21:407.

16. Chaitow L, Franke H, Fryer G, et al. Muscle energy techniques. 4th ed. Edinburgh: Churchill Livingstone/Elsevier; 2013.

17. Sharma D, Sen S. Effects of muscle energy technique on pain and disability in subjects with SI joint dysfunction. Int J Physiother Res 2014;2:305-11.

18. Hashemirad F, Karimi N, Keshavarz R. The effect of Kinesio taping technique on trigger points of the piriformis muscle. J Bodyw Mov Ther 2016;20:80714.

19. Alkady SM, Kamel RM, AbuTaleb E, Lasheen Y, Alshaarawy FA. Efficacy of mulligan mobilization versus muscle energy technique in chronic sacroiliac joint dysfunction. Int J Physiother 2017;4:311-8.

20. Van Leeuwen RJ, Szadek K, de Vet H, Zuurmond W, Perez R. Pain pressure threshold in the region of the sacroiliac joint in patients diagnosed with sacroiliac joint pain. Pain Physician 2016;19:147-54.

21. Added MA, de Freitas DG, Kasawara KT, Martin RL, Fukuda TY. Strengthening the gluteus maximus in subjects with sacroiliac dysfunction. Int J Sports Phys Ther 2018;13:114-20.
22. Copay AG, Cher DJ. Is the Oswestry Disability Index a valid measure of response to sacroiliac joint treatment? Qual Life Res 2016;25:283-92.

23. Wilson V, Douris P, Fukuroku T, Kuzniewski M, Dias J, Figueiredo P. The immediate and long-term effects of kinesiotape(R) on balance and functional performance. Int J Sports Phys Ther 2016;11:247-53.

24. Lee JH, Yoo WG, Kim MH, Oh JS, Lee KS, Han JT. Effect of posterior pelvic tilt taping in women with sacroiliac joint pain during active straight leg raising who habitually wore high-heeled shoes: a preliminary study. J Manipulative Physiol Ther 2014;37:2608.

25. Jonely H, Brismee JM, Desai MJ, Reoli R. Chronic sacroiliac joint and pelvic girdle dysfunction in a 35-year-old nulliparous woman successfully managed with multimodal and multidisciplinary approach. J Man Manip Ther 2015;23:20-6.

26. Visser LH, Woudenberg NP, de Bont J, et al. Treatment of the sacroiliac joint in patients with leg pain: a randomized-controlled trial. Eur Spine J 2013;22:2310-7.

27. Orakifar N, Kamali F, Pirouzi S, Jamshidi F. Sacroiliac joint manipulation attenuates alpha-motoneuron activity in healthy women: a quasi-experimental study. Arch Phys Med Rehabil 2012;93:56-61.

28. Levangie PK. Four clinical tests of sacroiliac joint dysfunction: the association of test results with innominate torsion among patients with and without low back pain. Phys Ther 1999;79:1043-57. 